\title{
Understanding the influence of left ventricular assist device inflow cannula alignment and the risk of intraventricular thrombosis
}

\author{
Michael Neidlin ${ }^{* *}$ (D), Sam Liao 1,2,3,4, Zhiyong Li ${ }^{4}$, Benjamin Simpson5, David M. Kaye ${ }^{3}$, Ulrich Steinseifer ${ }^{1,2}$ and \\ Shaun Gregory ${ }^{2,3}$
}

*Correspondence:

neidlin@ame.rwth-aachen.de

${ }^{1}$ Department

of Cardiovascular

Engineering, Institute

of Applied Medical

Engineering, Helmholtz

Institute, RWTH Aachen

University, Pauwelsstraße 20,

52074 Aachen, Germany

Full list of author information

is available at the end of the

article

\begin{abstract}
Background: Adverse neurological events associated with left ventricular assist devices (LVADs) have been suspected to be related to thrombosis. This study aimed to understand the risks of thrombosis with variations in the implanted device orientation. A severely dilated pulsatile patient-specific left ventricle, modelled with computational fluid dynamics, was utilised to identify the risk of thrombosis for five cannulation angles. With respect to the inflow cannula axis directed towards the mitral valve, the other angles were $25^{\circ}$ and $20^{\circ}$ towards the septum and $20^{\circ}$ and $30^{\circ}$ towards the free wall.
\end{abstract}

Results: Inflow cannula angulation towards the free wall resulted in longer blood residence time within the ventricle, slower ventricular washout and reduced pulsatility indices along the septal wall. Based on the model, the ideal inflow cannula alignment to reduce the risk of thrombosis was angulation towards the mitral valve and up to parallel to the septum, avoiding the premature clearance of incoming blood.

Conclusions: This study indicates the potential effects of inflow cannulation angles and may guide optimised implantation configurations; however, the ideal approach will be influenced by other patient factors and is suspected to change over the course of support.

Keywords: Heart failure, Cardiomyopathy, Cardiovascular surgery, Thrombosis, Computational fluid dynamics, Left ventricular assist device, Cannula position

\section{Background}

Despite favourable outcomes and technological advances of left ventricular assist devices (LVADs), many complications still exist [1]. The source of thromboemboli responsible for ischaemic strokes may originate from a number of sites [2-4]. Nonphysiological intraventricular flow patterns generated by the LVAD inflow cannula can be a contributing factor to thrombosis $[1,5-8]$. However, a common clinical goal for included in the article's Creative Commons licence and your intended use is not permitted by statutory regulation or exceeds the permitted use, you will need to obtain permission directly from the copyright holder. To view a copy of this licence, visit http://creativecommons.org/ licenses/by/4.0/. The Creative Commons Public Domain Dedication waiver (http://creativecommons.org/publicdomain/zero/1.0/) applies to the data made available in this article, unless otherwise stated in a credit line to the data. 
LVAD cannula placement is the prevention of occlusion and is not typically oriented to minimise flow-induced intraventricular thrombosis [9-11]. Numerical studies have suggested that thrombosis can be affected by a range of variables including, surgical technique to patient-specific anatomy and pump orientation [12, 13]. Clinical reports have highlighted the significance of the LVAD inflow cannula angle. For instance, the risk of HeartMate II pump thrombosis increases, and the rate of survival decreases if the angle between the inflow cannula and centre of the LVAD rotor is acute $\left(<55^{\circ}\right)[10,14]$. In particular, thrombosis was observed when the inflow cannula of a HeartMate II was directed towards the septum, which was attributed to increased suction events (leftward septal deviation leading to direct suction of the septum into the inflow cannula) and unfavourable intraventricular flows [15].

There have been limited studies assessing inflow cannula positioning; however, numerical studies have been undertaken [16]. A generic inflow cannula was apically implanted at angles of $-14^{\circ},-7^{\circ}, 0^{\circ},+7^{\circ},+14^{\circ}$ relative to the left ventricular long axis. Utilising a static left ventricle with a non-existent mitral valve, a Lagrangian approach (injection of discrete particles) was implemented to assess the risk of thrombosis. It was reported that cannulation angles between $\pm 7^{\circ}$ reduced the risk of thrombosis, based on reduced accumulated shear stress and residence time.

Clinical evaluation of LVAD inflow cannulation angles has typically been performed retrospectively, often as a result of unintended pump migration or mispositioning: conclusions are formed based on symptomatic clinical observations in contrast to understanding the problem. To better understand the relationship between LVAD inflow cannulation angle and thrombosis potential, this study addresses some limitations of previous numerical models such as neglecting the mitral valve, use of a rigid heart wall and the use of particles to evaluate the risk of thrombosis.

The presented study aimed to evaluate the risk of thrombosis in a pulsatile patientspecific left ventricle with variations in inflow cannula angles. Based on prior understandings of intraventricular flow under LVAD support [6, 7], it was hypothesised that a cannulation angle that promoted natural intraventricular flow patterns would be beneficial to reduce the risk of thrombosis. Five metrics were implemented to assess the risk of thrombosis: blood residence time, left ventricular washout, kinetic energy, instantaneous blood stagnation and pulsatility indices $[3,17,18]$.

\section{Results}

\section{Intraventricular flow dynamics under LVAD support}

Intraventricular velocity contours for early diastole and mid-diastole as well as early and end-systole are shown in Fig. 1. Cannulation towards the septum was at $25^{\circ}$ and $20^{\circ}$, assigned as $25 \mathrm{~s}$ and $20 \mathrm{~s}$, respectively. Angulation towards the free/lateral wall at $20^{\circ}$ and $30^{\circ}$ were assigned as $20 \mathrm{f}$ and $30 \mathrm{f}$. The $20^{\circ}$ angulation to either side was chosen to emphasise the effects of inflow cannula alignments. All cannulation angles resulted in a diastolic vortex despite severe angulation towards the free wall. Inflow cannula angulation towards the free wall appeared to guide the incoming flow towards the inflow cannula tip, as shown in early diastole and systole in Fig. 1. At end-systole, cannulation angles towards the septum had greater uniformity of velocity vectors that contributed 


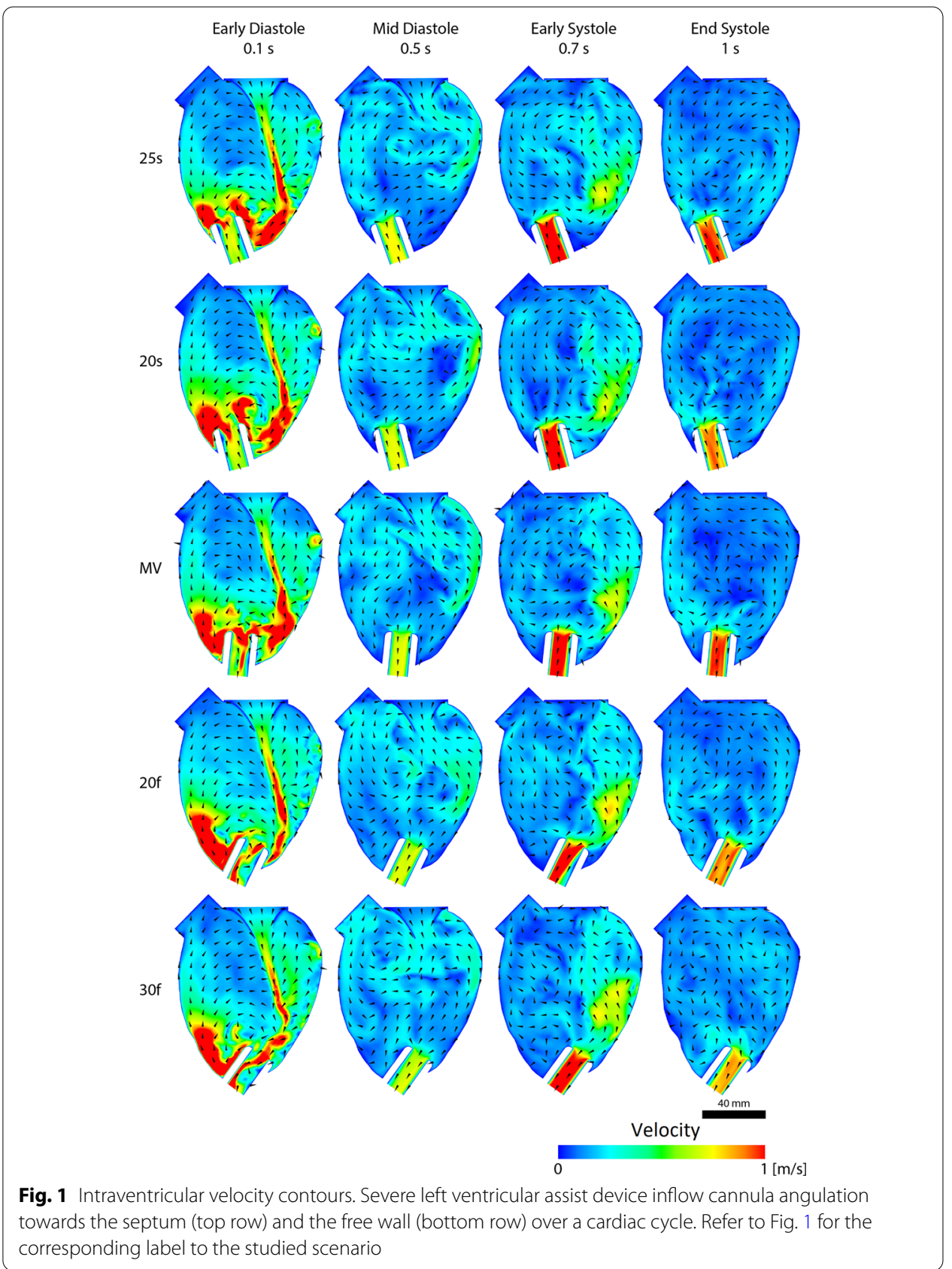

to a larger clockwise vortex, whereas cannulation angles towards the free wall showed multiple small clockwise vortices.

\section{Blood residence times}

Average blood residence times in the left ventricle were higher when the inflow cannula was angled towards the free wall, as shown in Table 1. In reference to the MV case (MV); $25 \mathrm{~s}, 20 \mathrm{~s}, 20 \mathrm{f}$ and $30 \mathrm{f}$ had a difference of $0.36 \%,-0.58 \%, 2.43 \%$ and $4.68 \%$, respectively. While minor angling the inflow cannula $20^{\circ}$ towards the septum was found to have the lowest blood residence time while further angulation to $25^{\circ}$ increased blood residence 
Table 1 Quantitative metrics to predict the risk of thrombosis

\begin{tabular}{|c|c|c|c|c|c|}
\hline \multirow[t]{2}{*}{ Analysis metric } & \multicolumn{5}{|l|}{ Models } \\
\hline & $25 \mathrm{~s}$ & $20 \mathrm{~s}$ & MV & $20 f$ & $30 f$ \\
\hline Blood residence time $[\mathrm{s}]$ & 6.66 & 6.60 & 6.64 & 6.80 & 6.95 \\
\hline Ventricular washout after four cardiac cycles [\%] & 56.81 & 56.78 & 57.81 & 58.09 & 58.29 \\
\hline Peak kinetic energy density of left ventricle $\left[\mathrm{J} / \mathrm{m}^{3}\right]$ & 326.04 & 325.74 & 328.42 & 325.91 & 324.67 \\
\hline
\end{tabular}

times. The basal regions, the regions around the mitral and aortic valves, had the longest blood residence times. Long blood residence times did not only occur around the left ventricular outflow tract, but also at the anterior and posterior aspects of the basal region, as shown in Fig. 2.

\section{Ventricular washout and kinetic energy}

The rate of ventricular washout after four cardiac cycles increased when the inflow cannula was angled towards the septum, as seen in Fig. 3 and Table 1. The largest difference after four cardiac cycles was $1.51 \%$. The variations in the alignment of incoming blood into the inflow cannula can also be seen in the Additional file 1: Fig. S1. The kinetic energy densities throughout the cardiac cycle are shown in Fig. 4 and the peak values are shown in Table 1. It can be seen that different cannula angulation does not influence either the peak kinetic energy density or the cycle-dependent energy density, with only negligible differences in the diastole.

\section{Pulsatility index}

The pulsatility index contour plot (Fig. 5) indicated that if the cannula was aligned towards the septum, a low pulsatility index (PI) was found at the left ventricular outflow tract $(\mathrm{PI}<1)$. Similarly, if the angulation was in close apposition to the free wall, then a low pulsatility index also occurs at the left ventricular outflow tract. Cannula alignment towards the mitral valve and free wall both had greater pulsatility indices at the left ventricular outflow tract (PI 2-3). The relative thickness of high pulsatility indices along the septal wall appeared to decrease as cannulation alignment rotated towards the free wall.

\section{Discussion}

The risk of intraventricular thrombosis due to changes in inflow cannula cannulation angles implanted at the left ventricular apex was investigated. Based on the modelled scenario, this study determined whether the common cannula alignment towards the mitral valve is best practice and evaluated potential consequences when deviations occurred. We used a numerical approach going beyond the existing computational models considering ventricular wall movement, a mitral valve geometry and the surrounding cardiovascular system by means of a lumped parameter network. Results based on the blood residence time, rate of left ventricular washout, kinetic energy and pulsatility indices elucidated that inflow cannula alignment towards the mitral valve resulted in potentially more favourable flow dynamics than severe angulation towards either the septum or free wall. 


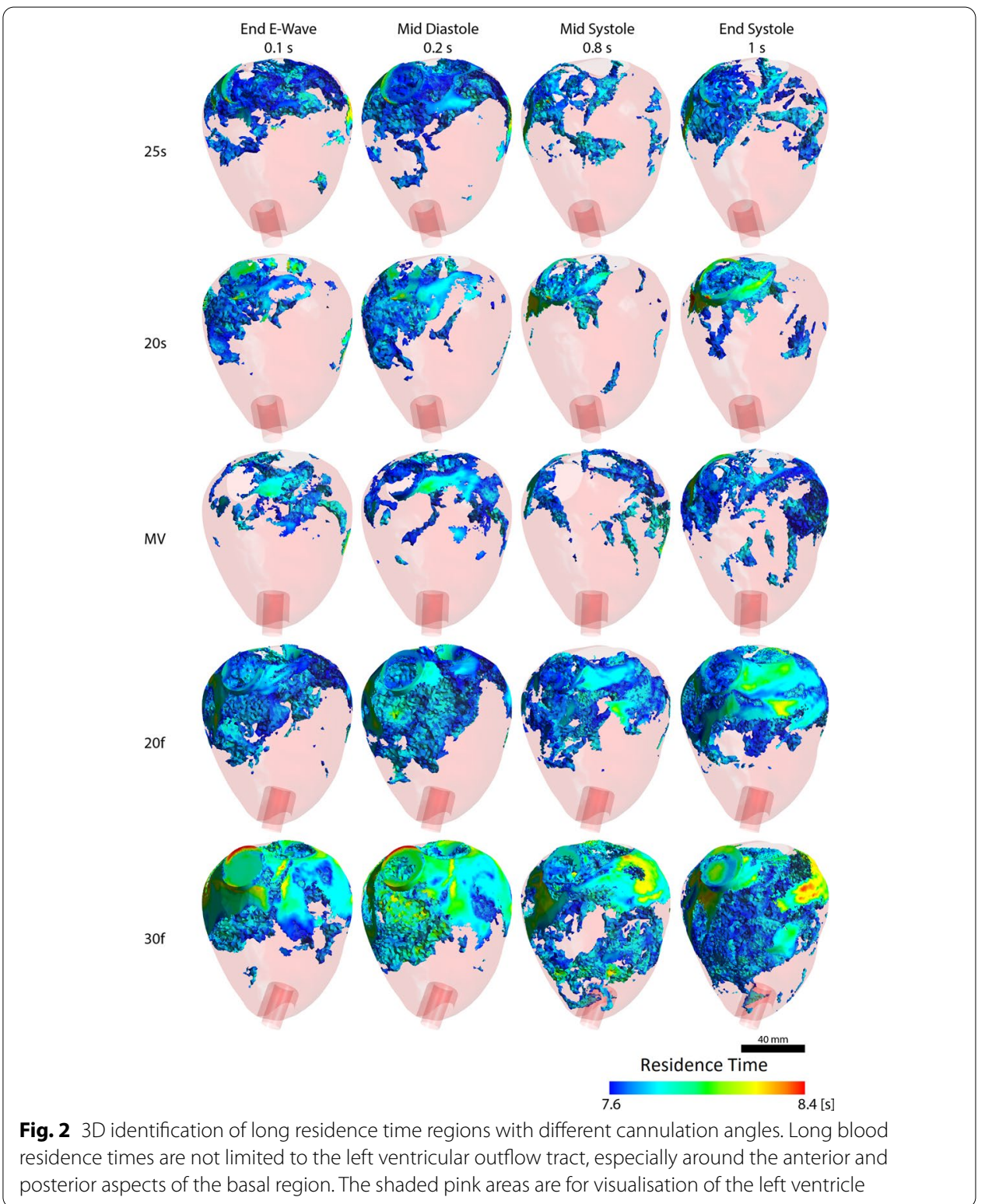

From clinical observations of acute angles between the HeartMate II pump body and inflow cannula, it was difficult to determine the exact position of the inflow cannula relative to internal left ventricular features from the presented literature $[10,14,19]$. Nevertheless, this study assumed that an acute inflow cannula to pump angle might have the highest probability of being directed towards the septum. The increased risk of pump thrombosis due to this angulation could be correlated with the $25 \mathrm{~s}$ case, whereby the total blood residence time was $0.8 \%$ higher than inflow cannula angulation towards the mitral valve. However, angulation towards the free wall was found to be worse than septal alignment as blood residence times were up to $5 \%$ longer than mitral valve alignment. Considering the small differences, the clinical observation of increased pump thrombosis may not be solely attributed to the inflow 


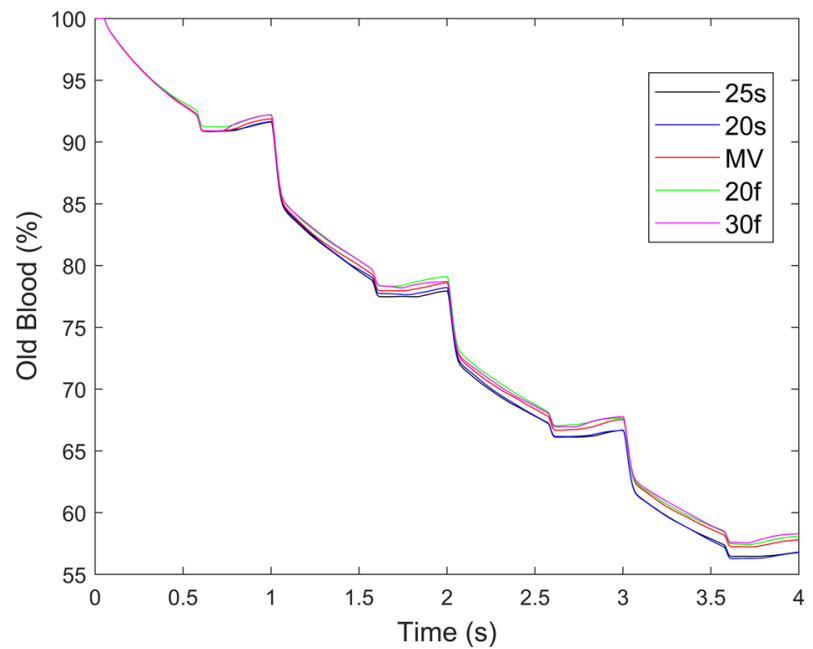

Fig. 3 Rate of ventricular washout with different cannula angles. The rate of washout increased with angulation towards the septum. Ventricular washout describes the rate that old blood within the left ventricle is replaced with incoming blood from the left atrium

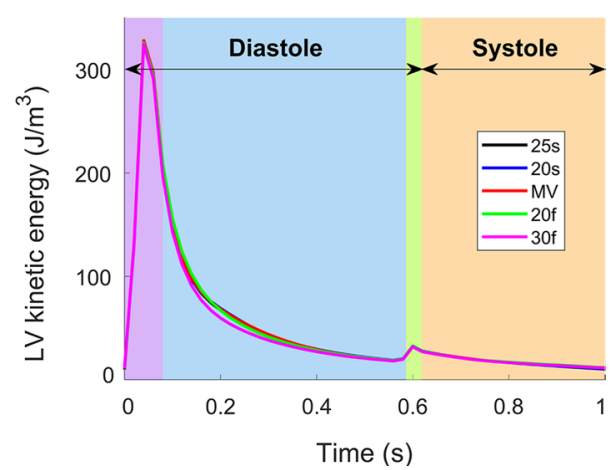

Fig. 4 Mean left ventricular kinetic energy density of four cardiac cycles. The purple, blue, green and orange shaded areas correspond to the E-wave, mid-diastole, A-wave and systole, respectively

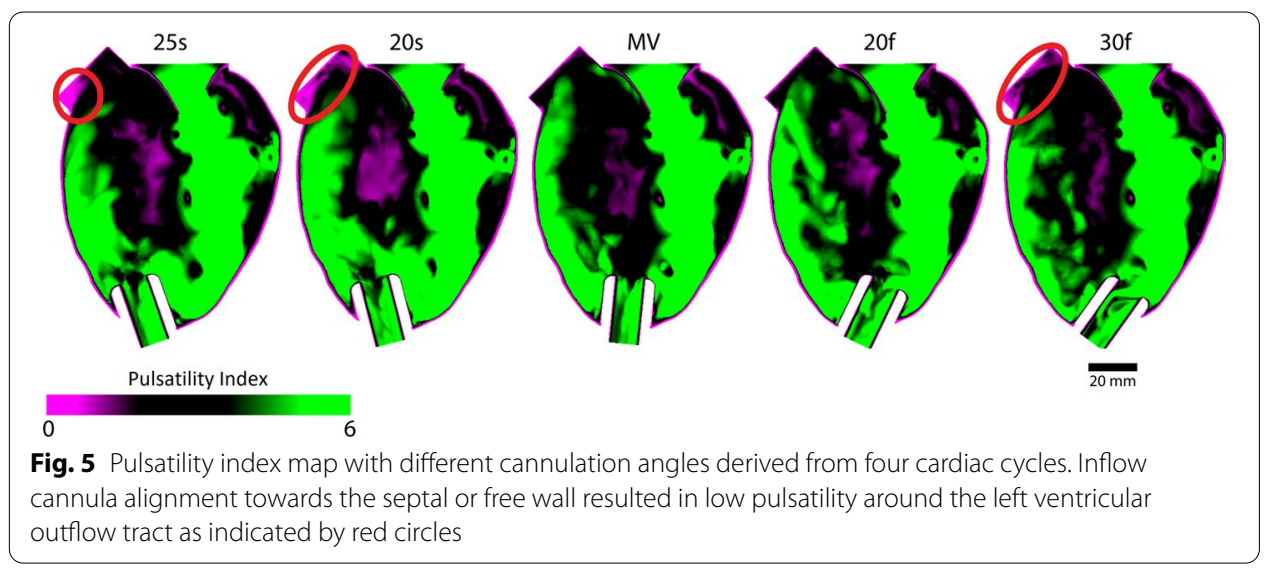


cannula angle regarding the total blood residence time; however, it may have a cumulative effect with other factors. These include the biochemical elements of thrombus formation such as platelet activation by tissue factor and the role of the coagulation cascade [20]. Further aspects are the cannula design and insertion length as well as clinically relevant parameters like excessive LVAD speed, LV size and lack of aortic valve opening [16].

Longer blood residence times were consistently observed around the left ventricular outflow tract, but with cannula angulation towards the free wall, the LV anterior and posterior basal regions had increased volumes of prolonged residence times, highlighting the importance of assessing the entire left ventricular volume and not only in a single plane or section $[8,21]$. Despite arguably small differences in blood residence times $(\sim 5 \%)$ and the rate of washout $(\sim 1 \%)$, it provided an interesting perception of the effects of cannulation angles. Even the slightest improvement to intraventricular flow could be advantageous considering that small thrombi may be conducive for the formation of larger structures due to a cumulative effect $[3,22]$.

Orientating the inflow cannula towards the free wall may increase the probability of inducing shorter flow paths between the left atrium and inflow cannula tip. The idea to increase the flow path between the mitral valve and inflow cannula tip, to preserve the natural clockwise vortex, has the potential to reduce the risk of thrombosis $[6,7]$. Even though alignment towards the septum was more favourable than the free wall, if the inflow cannula long axis intersected the septum, then lower pulsatility indices were observed at the left ventricular outflow tract along with higher blood residence times. Reduced pulsatility indices for when the inflow cannula is angled towards the septum has been previously reported in a PIV study [23]. Based on the results, it is recommended to avoid alignment of the inflow cannula towards the septum, which would also avoid inflow cannula occlusion. The maximum angulation limit towards the septum could potentially be parallel to the septal wall, which may also prevent septal shift at higher pump speeds and reduce the likeliness of left ventricular suction, similar to suggestions by Chivukula et al. despite modelling differences [16].

Chivukula et al. indicated that when the inflow cannula was angled closer to alignment with the LV long axis, flow from the mitral valve to the inflow cannula was more direct [16]. However, this may not be typical for patients with dilated cardiomyopathy: the mitral jet is often directed more towards the free wall [24]. Furthermore, it was speculated that the direct flow between the mitral valve and inflow cannula inlet was favourable, contrary to the recommendations presented here. Due to the evaluation method of injected particles using a Lagrangian approach, the direct flow from the mitral valve to the inflow cannula does not entirely explain the risk of intraventricular thrombosis. Perhaps due to the smaller simulated left ventricular geometry, the effects may not be as pronounced in capturing recirculation zones, hence similar conclusions.

Attenuation of vortex structures due to cannulation orientation has similarly been reported [5]. The previous in vitro study suggested that when an inflow cannula was aligned towards the left ventricular outflow tract, more distinct vortices were formed, corroborating with the presented results in our study. Comparing the velocity vectors between $25 \mathrm{~s}$ and 30f in Fig. 1 at end-systole, a large uniform clockwise fluid rotation was present when the inflow cannula was angled towards the septum and mitral valve. 
In contrast, when the inflow cannula was angled towards the free wall, multiple smaller vortices were present.

The pulsatility index contour plot (Fig. 5) indicated that $25 \mathrm{~s}, 20 \mathrm{~s}$ and $30 \mathrm{f} \mathrm{had} \mathrm{low} \mathrm{indi-}$ ces around the left ventricular outflow tract, which can be related to the blood residence time visualisation in Fig. 2. Interestingly, the pulsatility index contour plot of the mitral jet can also be associated with the rate of ventricular washout (Fig. 3): the high pulsatility regions due to the mitral jet can be seen to align with the inflow cannula, especially with angulation towards the free wall (20f and $30 \mathrm{f}$ ); therefore, potentially explaining the fast but detrimental clearance of incoming blood. The calculated pulsatility indices correspond with the results obtained in the in vitro study [3] with values between 0 and 6 throughout the left ventricle, values below 1 at the left ventricular outflow tract and values $>5$ in most parts of the left ventricle. It has to be noted, that pulsatility index is a spatially resolved value and, if the interest lies in obtaining a spatially averaged parameter, the blood residence time should be used instead.

The most significant finding in this study was in agreement with Laumen et al. [5]. There, it was suggested that the ideal inflow cannula placement would be parallel to the septal wall. It was thought that an inflow cannula position pointed towards the septal wall would result in a large uniform vortex, which was found in the presented study. Nevertheless, the alignment of inflow cannulae is not solely related to cannula angulation at the apex, but also the site of cannulation and patient-specific left ventricular geometry.

The results elucidated the importance of cannulation angles, emphasising the significance to minimise early clearance of incoming blood during diastole. However, consideration of endocardial contact and suction should not be overlooked. It is expected that no ideal inflow cannula angle, length or site exists for all patients, but instead will be dependent on the patient condition, such as the degree of left ventricular dilation.

\section{Limitations}

Limitations of the model have been previously described, which included the assumption of smooth endocardial walls; ventricular movement in a balloon-like manner based on reduced twist [25]; and neglected internal left ventricular features [26]. Laminar flow was assumed, however the flow might be in the transitional/turbulent regimen, necessitating turbulence modelling. The effect of this simplification on the thrombus risk metrics should be evaluated. The assumed heart rate of $60 \mathrm{bpm}$ is low for VAD patients and may not represent typical patients and only one patient-specific geometry was investigated. Thus, it must be remembered that this paper only presents one potential scenario and the results must be interpreted with caution. All the evaluated metrics indirectly predict the risk of thrombosis and are only an indicator of blood stagnation. A quantifiable relation of these haemodynamic parameters (blood residence time, washout, pulsatility index) towards thrombus formation if difficult to determine as spatially resolved experimental data on thrombus formation in the LV during LVAD support is scarce to non-existent. Each metric has its advantages and disadvantages. Blood residence time, ventricular washout and kinetic energy are spatially averaged metrics allowing for a quick comparison, however neglecting differences within the LV. On the other hand, pulsatility 
index and the ventricular flow field can be resolved spatially (and if required temporally), however a quantitative comparison for several cases might become difficult. Thus, rather a combination of these metrics should be considered when interpreting the results and we do not believe that there is one single value that can serve as a surrogate marker for flow-induced thrombus formation.

With the awareness of limitations intrinsic to numerical studies, the direct clinical significance is difficult to predict. However, further insights provided into intraventricular flow characteristics due to changes in cannulation angles may provide additional aspects of consideration when designing and implanting LVADs. Current clinical principles for inflow cannula alignment is to angle the inflow cannula parallel to the septum and towards the left ventricular centre, which is in agreement with the suggestions presented in this study [27]. However, the presented findings may be limited to the simulated patient condition; therefore, other haemodynamic and spatial combinations should be pursued. Since no aortic valve opening was simulated and atypical to clinical preference, it is hypothesised that superior ventricular washout could be obtained in the basal region if aortic valve opening is promoted; however, parallel septal alignment is expected to be superior based on the avoidance of early blood clearance and higher kinetic energies at the LV apex.

In order to leverage clinical significance, future applications of this numerical model should focus on a higher number of patient-specific geometries. One case study, as shown within this work, is not able to quantify the role of flow alterations and provide statistically significant results with a clinical implication. Investigations of many geometries require much higher computational costs, a problem that needs to be tackled as the next step. We believe that reduction of model complexity under simultaneous considerations of the thrombus risk metrics presented herein, should be performed. In other words, one should gradually simplify the model and compare the changes in the outcomes such as pulsatility index, blood residence time and intraventricular vortices. Possible simplifications could be steady-state simulations at some time points instead simulations of the full cardiac cycle, a rigid wall assumption instead of a fluid-structure interaction simulation or the disregard of the lumped parameter network model.

The results from our study can be used as a reference case to see, if model simplifications violate the prediction of numerical models focussing on thrombus risk in the left ventricle during LVAD support.

\section{Conclusions}

Understanding the effect of inflow cannulation angle on intraventricular flow is non-trivial in vivo; thus, this study endeavoured to provide insights into the effect of inflow cannulation angles on intraventricular flow numerically. Notwithstanding the model limitations, the ideal inflow cannula alignment, likely to be patient and device-dependent, should be positioned in a manner to prevent occlusion and the premature clearance of blood directly entering the left ventricle. The ideal inflow cannula alignment in this simulated patient condition was angled towards the mitral valve and up to parallel to the septum. 


\section{Methods}

The numerical simulation (Fluent, ANSYS 18.0, PA, USA) consisted of three coupled components: (1) a zero-dimensional lumped parameter network providing the boundary conditions to the computational fluid dynamics simulation; (2) a fictitious heart wall with a pressure loading to provide heart wall motion; and (3) a severely dilated patientspecific left ventricle for the three-dimensional computational fluid dynamics model. The coupling of these components have been extensively described elsewhere [6, 7]; however, a summary is provided here.

\section{Lumped parameter network model}

The cardiovascular system was represented in a zero-dimensional form by a series of ordinary differential and algebraic equations that were represented with ten compartments [6,28]. Each vessel compartment was described using a two-element (resistance and compliance) Windkessel model. The model simulated a heart failure condition under LVAD support by targeting a cardiac output of $5 \mathrm{~L} / \mathrm{min}$ and a mean aortic pressure between 75 and $90 \mathrm{mmHg}$. The LVAD was based on a HeartWare HVAD running at a constant speed of $2600 \mathrm{rpm}$. The transmitral flow calculated from the lumped parameter network model was one-way coupled to the 3D computational fluid dynamics model at the mitral valve inlet. A more detailed description of the lumped parameter network model together with the chosen parameters is included in the Additional file 1. Figure 6a shows the transmitral flow and the LVAD flow as computed by the lumped parameter network model.

\section{Heart wall motion}

The heart wall motion was simulated by loading a fictitious left ventricular wall with a time-dependent pressure profile, which was based on the left ventricular stroke volume
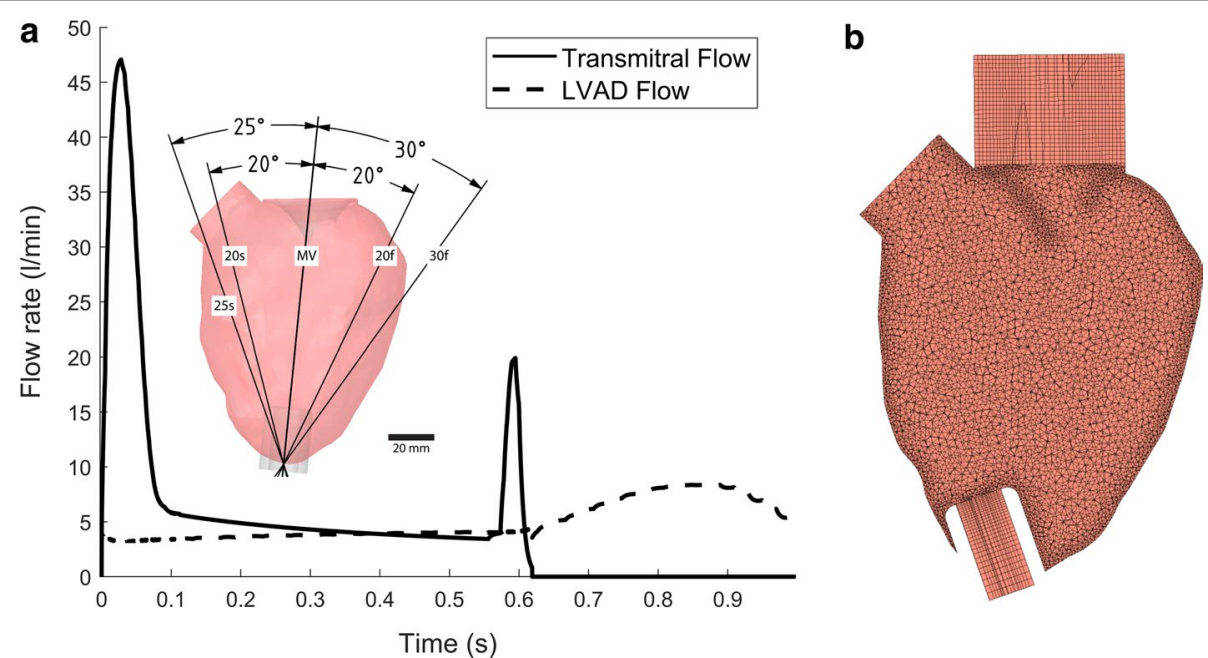

Fig. 6 Evaluated inflow cannula angles, boundary conditions and mesh. a The alignment of the inflow cannula towards the mitral valve was used as the reference case. The flow rates were driven by a lumped parameter network (LPN) that was one-way coupled to the computational fluid dynamics simulation. $\mathbf{b}$ Computational mesh for the 25 s scenario 
simulated from the lumped parameter network model, as previously presented [7]. The deformation of the wall was transferred to the mesh of the fluid domain in a one-way coupled manner.

\section{Left ventricular geometry}

The left ventricular geometry, reconstructed from computed tomography, was from an LVAD candidate with a severely dilated LV; see [7]. A parametric mitral valve was placed in the anatomical position with guidance from the medical imaging data. The mitral valve state was coupled to the lumped parameter network model which triggered the valve to be either on or off. Five different cannulation angles with an insertion length of $24 \mathrm{~mm}$ were evaluated (Fig. 6). The reference angle was defined to be when the inflow cannula was in alignment with the mitral valve, assigned as MV, as typically seen in the clinic [29]. Two angles to the septal $\left(25^{\circ}\right.$ and $\left.20^{\circ}-25 \mathrm{~s}, 20 \mathrm{~s}\right)$ and lateral sides ( $30^{\circ}$ and $\left.20^{\circ}-30 \mathrm{f}, 20 \mathrm{f}\right)$ angled on the coronal plane were studied. The difference in maximum angulation, $25 \mathrm{~s}$ vs. 30f, representing severe mispositioning between the two sides was restricted by the inflow cannula endocardial contact. For the simulated heart failure condition, the aortic valve did not open to simulate the worst-case scenario.

\section{Computational fluid dynamics model}

Meshing strategy and element sizes were chosen on previous mesh convergence studies (blood residence time and rate of washout were taken as metrics for mesh independence) [7]. The model had approximately one million cells with a max. skewness of 0.9 and min. mesh orthogonal quality of 0.28 . The ventricle was meshed with unstructured tetrahedral elements with a size of $2 \mathrm{e}-3 \mathrm{~m}$. The mesh was refined at the ventricular walls, the mitral valve and the cannula by enabling mesh defeaturing with a size of $5 e-4 \mathrm{~m}$. The inlet domain of the model and the inlet cannula were meshed with hexahedral elements with a size of $2 \mathrm{e}-3 \mathrm{~m}$ through sweeping operations. Figure $6 \mathrm{~b}$ shows the mesh for the $25 \mathrm{~s}$ case. The models were initialised for $30 \mathrm{~s}$, and then $4 \mathrm{~s}$ were simulated for the analysis of the results at a heart rate of 60 beats per minute. The density of blood was assumed to be $1060 \mathrm{~kg} / \mathrm{m}^{3}$. A Carreau model was used to model the non-Newtonian behaviour of blood [30]. Laminar flow was assumed, as the average Reynolds number was below 1000 as measured at the mitral inlet. Existence of turbulence has been a debate and other CFD studies have also assumed laminar flow [31, 32]. The PISO algorithm was used to solve the Navier-Stokes equations. Temporal discretization was performed with a second-order implicit scheme. Spatial discretization for the momentum was second-order upwind and for the pressure the pressure staggering option was selected. The deformation of the fluid mesh due to the heart wall motion was treated with diffusion-based smoothing to prevent the collapse of the mesh elements. The scaled convergence criteria were set to $10-5$ for $x / y / z$ velocity and continuity. The total CPU time was 6240 corehours per model, excluding initialisation, with an Intel Xeon $2.5 \mathrm{GHz}$ processor.

\section{Analysis of thrombus risk}

Currently, there is no gold-standard for the numerical prediction of thrombosis; however, various metrics have been previously utilised to predict the risk of thrombosis based on the concept of blood stasis. Therefore, four metrics have been implemented 
to assess the risk of thrombosis, including blood residence time, ventricular washout, kinetic energy and the pulsatility index [3, 17-19]. Our previous studies [6, 7] explain the implementation in more detail.

Regions of relatively stagnant blood can be evaluated by the time that blood resides within the left ventricle. The blood residence time was evaluated with an Eulerian approach, whereby all domains were initialised to $0 \mathrm{~s}$. Even though no diffusion was assumed, the Eulerian approach intrinsically has numerical diffusion. The simulations were initialised until the blood residence time reached a steady state with each cardiac cycle. The mean blood residence time in the left ventricular volume was timeaveraged over four cycles. The blood residence time analysis provides a global insight into the blood residence time dynamics.

Closely related to blood residence time, ventricular washout is the rate of replacing old blood with "fresh" incoming blood from the left atrium into the left ventricle [17]. The difference between blood residence time and ventricular washout is that ventricular washout provides insights into the time-dependent flow paths. A fast rate of ventricular washout could reduce the risk of thrombosis by reducing blood stasis. Ventricular washout was simulated using a virtual ink concept. All fluid domains were initialised with an ink concentration of 0 and the incoming ink 1, representing fresh blood entering from the left atrium. The identification of incoming blood was activated after flow initialisation. The percentage of old blood in the ventricle overtime was normalised by the left ventricular volume.

High kinetic energy within the left ventricle may be beneficial to reduce the risk of thrombosis due to increased blood motion and kinetic energy can be connected to the functional performance of the ventricle [33]. The cycle-dependent kinetic energy was calculated from the volume-averaged dynamic pressure and averaged over four cycles.

The pulsatility index indicates the maximum flow velocity variations in a localised area, where high pulsatility indices may signify greater acceleration/deceleration of blood, thus a lower probability of blood stasis [3]. The pulsatility index evaluated the difference between the maximum and minimum velocity of each pixel in the plane of interest over four cardiac cycles at a sample rate of $0.02 \mathrm{~s}$. The difference was normalised by the volume-average mean velocity over the cardiac cycles and was calculated according to Eq. (1):

$$
\mathrm{PI}=\frac{\mathrm{v}_{\max }-\mathrm{v}_{\min }}{\mathrm{v}_{\mathrm{ave}}} .
$$

\section{Supplementary Information}

The online version contains supplementary material available at https://doi.org/10.1186/s12938-021-00884-6.

Additional file 1: Includes supplementary methods and results.

Acknowledgements

Computational resources and services used in this work were provided by the High-Performance Computing and Research Support Group, Queensland University of Technology, Brisbane, Australia. 
the result interpretation and the relation to the clinical context. MN and SL drafted the manuscript with the input of all co-authors. SG and US supervised the execution of the entire study. All authors read and approved the final manuscript.

\section{Funding}

Open Access funding enabled and organized by Projekt DEAL. The authors would like to recognise the financial assistance by the Research Training Program, The Prince Charles Hospital Foundation (MS2013-29, PRO2014-08, TM2017-04), the National Health and Medical Research Council of Australia (APP1079421). Shaun D Gregory (102062) and Sam Liao (102499) were supported by Fellowships from the National Heart Foundation of Australia.

\section{Availability of data and materials}

Available upon request.

\section{Declarations}

Ethics approval and consent to participate

Ethics approval for use of patient images is present.

\section{Consent for publication}

Not applicable.

\section{Competing interests}

The authors declare that they have no competing interests.

\section{Author details}

${ }^{1}$ Department of Cardiovascular Engineering, Institute of Applied Medical Engineering, Helmholtz Institute, RWTH Aachen University, Pauwelsstraße 20, 52074 Aachen, Germany. ${ }^{2}$ Department of Mechanical and Aerospace Engineering, Monash University, Clayton, VIC 3800, Australia. ${ }^{3}$ Cardio-Respiratory Engineering and Technology Laboratory (CREATElab), Baker Heart and Diabetes Institute, Melbourne, VIC 3004, Australia. ${ }^{4}$ Institute of Health and Biomedical Innovation (IHBI), Queensland University of Technology (QUT), Kelvin Grove, QLD 4059, Australia. ${ }^{5}$ Department of Engineering, Nottingham Trent University, Clifton Lane, Nottingham NG11 8NS, UK.

Received: 25 February 2021 Accepted: 30 April 2021

Published online: 11 May 2021

\section{References}

1. Kirklin J, Pagani F, Kormos R, Stevenson L, Blume E, Myers S, et al. Eighth annual INTERMACS report: special focus on framing the impact of adverse events. J Heart Lung Transplant. 2017;36(10):1080-6.

2. Kurihara C, Ono M, Nishimura T, Saito A, Taketani T, Hisagi M, et al. Use of DuraHeart ${ }^{\circledR}$ support for more than 1 year as the first successful bridge to heart transplantation in Japan. J Artif Organs. 2010;14:67-9.

3. Reider C, Moon J, Ramesh V, Montes R, Campos J, Herold B, et al. Intraventricular thrombus formation in the LVADassisted heart studied in a mock circulatory loop. Meccanica. 2017;52:515-28.

4. Estep J, Stainback R, Little S, Torre G, Zoghbi W. The role of echocardiography and other imaging modalities in patients with left ventricular assist devices. JACC Cardiovasc Imag. 2010;3(10):1049-64.

5. Laumen M, Kaufmann T, Timms D, Schlanstein P, Jansen S, Gregory S, et al. Flow analysis of ventricular assist device inflow and outflow cannula positioning using a naturally shaped ventricle and aortic branch. Artif Organs. 2010;34(10):798-806.

6. Liao S, Wu E, Neidlin M, Li Z, Simpson B, Gregory S. The influence of rotary blood pump speed modulation on the risk of intraventricular thrombosis. Artif Organs. 2018;42:943-53.

7. Liao S, Neidlin M, Li Z, Simpson B, Gregory S. Ventricular flow dynamics with varying LVAD inflow cannula lengths: in-silico evaluation in a multiscale model. J Biomech. 2018;72:106-15.

8. May-Newman K, Vu V, Herold B. Modeling the link between left ventricular flow and thromboembolic risk using lagrangian coherent structures. Fluids. 2016;1:38.

9. Slaughter M, Pagani F, Rogers J, Miller L, Sun B, Russell S, et al. Clinical management of continuous-flow left ventricular assist devices in advanced heart failure. J Heart Lung Transplant. 2010;29(4 Suppl):S1-39.

10. Taghavi S, Ward C, Jayarajan S, Gaughan J, Wilson L, Mangi A. Surgical technique influences HeartMate II left ventricular assist device thrombosis. Ann Thorac Surg. 2013;96(4):1259-65.

11. Carr C, Jacob J, Park S, Karon BL, Williamson E, Araoz P. CT of left ventricular assist devices. Radiogr Rev Publ Radiol Soc N Am. 2010;30(2):429-44.

12. Girdhar G, Xenos M, Alemu Y, Chiu W-C, Lynch BE, Jesty J, et al. Device thrombogenicity emulation: a novel method for optimizing mechanical circulatory support device thromboresistance. PLoS ONE. 2012;7:e32463.

13. Chiu WC, Tran P, Khalpey Z, Lee E, Woo Y-R, Slepian M, Bluestein D. Device thrombogenicity emulation: an in silico predictor of in vitro and in vivo ventricular assist device thrombogenicity. Sci Rep. 2019;9.

14. Kazui T, Zhang A, Greenberg JW, Itoh A, Tran P, Keith A, et al. Left ventricular assist device inflow angle and pump positional change over time adverse impact on left ventricular assist device function. Annal Thorac Surg. 2016;102(6):1933-40.

15. Sorensen E, Kon Z, Feller E, Pham S, Griffith B. Quantitative assessment of inflow malposition in two continuous-flow left ventricular assist devices. Ann Thorac Surg. 2018;105(5):1377-83.

16. Chivukula V, Beckman J, Prisco A, Dardas T, Lin S, Smith J, et al. Left ventricular assist device inflow cannula angle and thrombosis risk. Circul Heart Fail. 2018;11:e004325. 
17. Sonntag S, Kaufmann T, Büsen M, Laumen M, Gräf F, Linde T, Steinseifer U. Numerical washout study of a pulsatile total artificial heart. Int J Artif Organs. 2014;37:241-52

18. Karches T, Buzás K. Methodology to determine residence time distribution and small scale phenomena in settling tanks. WIT Trans Eng Sci. 2011;70:117-25.

19. Fraser K, Zhang T, Taskin M, Griffith B, Wu Z. Computational fluid dynamics analysis of thrombosis potential in left ventricular assist device drainage cannulae. ASAIO J. 2010;56:157-63.

20. Rojano RM, Zhussupbekov M, Antaki J. Multi-constituent simulation of thrombus formation at LVAD inlet cannula connection: Importance of Virchow's triad. Artif Organs. 2021.

21. Hendabadi S, Bermejo J, Benito Y, Yotti R, Fernández-Avilés F, Álamo JD, Shadden S. Topology of blood transport in the human left ventricle by novel processing of doppler echocardiography. Ann Biomed Eng. 2013;41:2603-16.

22. May-Newman K, Wong Y, Adamson R, Hoagland P, Vu V, Dembitsky W. Thromboembolism is linked to intraventricular flow stasis in a patient supported with a left ventricle assist device. ASAIO J. 2013;59:452-5.

23. May-Newman K, Marquez-Maya N, Montes R, Salim S. The effect of inflow cannula angle on the intraventricular flow field of the left ventricular assist device-assisted heart: an in vitro flow visualization study. ASAIO J. 2019;65:139-47.

24. Chan BT, Yeoh HK, Liew Y, Aziz YFA, Sridhar GS, Hamilton-Craig C, et al. Left ventricular flow propagation velocity measurement: is it cast in stone? Med Biol Eng Comput. 2017;55:1883-93.

25. Sengupta P, Tajik A, Chandrasekaran K, Khandheria B. Twist mechanics of the left ventricle: principles and application. JACC Cardiovasc Imaging. 2008;1 (3):366-76.

26. Vedula V, Seo JH, Lardo A, Mittal R. Effect of trabeculae and papillary muscles on the hemodynamics of the left ventricle. Theoret Comput Fluid Dyn. 2016;30:3-21.

27. Adamson R, Mangi A, Kormos R, Farrar D, Dembitsky W. Principles of HeartMate II implantation to avoid pump malposition and migration. J Card Surg. 2015;30:296-9.

28. Neidlin M, Corsini C, Sonntag S, Schulte-Eistrup S, Schmitz-Rode T, Steinseifer U, et al. Hemodynamic analysis of outflow grafting positions of a ventricular assist device using closed-loop multiscale CFD simulations: preliminary results. J Biomech. 2016;49(13):2718-25.

29. Slaughter M. Implantation of the HeartWare left ventricular assist device. Semin Thorac Cardiovasc Surg. 2011;23(3):245-7.

30. Johnston BM, Johnston P, Corney S, Kilpatrick D. Non-Newtonian blood flow in human right coronary arteries: steady state simulations. J Biomech. 2004;37(5):709-20.

31. Arefin MS, Morsi Y. Fluid structure interaction (FSI) simulation of the left ventricle (LV) during the early filling wave (E-wave), diastasis and atrial contraction wave (A-wave). Australas Phys Eng Sci Med. 2014;37:413-23.

32. Zając J, Eriksson J, Dyverfeldt P, Bolger A, Ebbers T, Carlhäll C-J. Turbulent kinetic energy in normal and myopathic left ventricles.J Magn Reson Imag. 2015:41:1021-9.

33. Seo JH, Mittal R. Effect of diastolic flow patterns on the function of the left ventricle. Phys Fluids. 2013;25:110801.

\section{Publisher's Note}

Springer Nature remains neutral with regard to jurisdictional claims in published maps and institutional affiliations.

- fast, convenient online submission

- thorough peer review by experienced researchers in your field

- rapid publication on acceptance

- support for research data, including large and complex data types

- gold Open Access which fosters wider collaboration and increased citations

- maximum visibility for your research: over 100M website views per year

At BMC, research is always in progress.

Learn more biomedcentral.com/submissions 